\title{
Prescripción de anticoagulación oral en el paciente geriátrico con fibrilación auricular
}

\author{
Prescription of oral anticoagulation in geriatric patients with atrial fibrillation
}

Génesis Ramírez-Prieto ${ }^{1}$, José E. Pombo-Bartelt², Guadalupe Rojas-Calderón ${ }^{3}$ y

José J. García-González ${ }^{3 *}$

${ }^{1}$ Departamento de Geriatría, Hospital General de Zona N. ํ 67, Instituto Mexicano del Seguro Social, N.L.; '2Departamento de Cardiología, Instituto Corazón de Querétaro y Hospital Ángeles de Querétaro, Qro.; ${ }^{3}$ Departamento de Geriatría, Hospital General Regional N.ำ 1, Instituto Mexicano del Seguro Social, Qro. México

\section{Resumen}

Objetivo: Determinar la prevalencia de prescripción de anticoagulación oral en pacientes > 60 años con fibrilación auricular no valvular (FANV). Métodos: Estudio observacional, transversal, retrospectivo en el que se revisaron los expedientes de pacientes > 60 años que acudieron a la consulta externa de especialidades (cardiología, medicina interna, geriatría) de un hospital de segundo nivel de atención en Querétaro, México, con el diagnóstico de FANV del 1 de julio al 30 de septiembre de 2019. Se analizaron el perfil clínico y el tratamiento anticoagulante. Resultados: Se incluyeron 300 pacientes (edad media: $77.2 \pm 8.3$ años; 53.3\% mujeres; $81.0 \%$ atendidos en cardiología). El 91\% presentaban un riesgo tromboembólico elevado, el $22.7 \%$ un riesgo hemorrágico elevado y el 1.7\% contraindicaciones para la anticoagulación. La presencia de comorbilidades fue frecuente. El $82.7 \%$ estaban tomando anticoagulantes orales de acción directa (ACOD), el 11.0\% antagonistas de la vitamina $K(A V K)$, y el 6.3\% no estaban tomando ningún tratamiento anticoagulante. El 29.3\% de los pacientes estaban tomando anticoagulantes orales de manera inadecuada, siendo las dos principales causas la prescripción de dosis de ACOD no ajustada a edad, peso y nivel de creatinina y la administración de ACOD sin indicación de acuerdo con el riesgo tromboembólico. De los pacientes que tomaban AVK, solo el 39.4\% presentaban una anticoagulación en rango terapéutico. De los tratados con ACOD, el 48.0\% tomaban rivaroxabán, principalmente con una dosis de 20 mg/día (73.1\%). Conclusiones: El riesgo tromboembólico en pacientes geriátricos con FANV es elevado. En menos del 2\% existe una contraindicación para la anticoagulación. En tres de cada diez pacientes se prescriben de manera inadecuada los anticoagulantes orales.

Palabras clave: Geriátrico. Antagonistas de la vitamina K. Anticoagulación. Anticoagulantes orales de acción directa. Fibrilación auricular.

\footnotetext{
Abstract

Objective: To determine the prevalence of prescription of oral anticoagulation in patients aged > 60 years with nonvalvular atrial fibrillation (NVAF). Methods: Observational, cross-sectional, retrospective study based on a review of the clinical histories of patients aged >60 years diagnosed with NVAF from July 1 to September 30, 2019 and seen at the outpatient clinic (cardiology, internal medicine, geriatrics) of a secondary-level hospital in Queretaro, Mexico. Clinical profile and oral anticoagulant

Correspondencia:

*José J. García-González

E-mail: josejuangarciaglz@yahoo.com.mx

Disponible en internet: 18-06-2021 Arch Cardiol Mex. 2022;92(1):42-52 www.archivoscardiologia.com

1405-9940 / @ 2021 Instituto Nacional de Cardiología Ignacio Chávez. Publicado por Permanyer. Este es un artículo open access bajo la licencia CC BY-NC-ND (http://creativecommons.org/licenses/by-nc-nd/4.0/).

Fecha de recepción: 16-12-2020

Fecha de aceptación: 14-04-2021 DOI: 10.24875/ACM.20000563
} 
treatment were analyzed. Results: The study population comprised 300 patients (mean age, $77.2 \pm 8.3$ years; $53.3 \%$ women; $81 \%$ attended in cardiology). Of these, $91 \%$ had a high thromboembolic risk, $22.7 \%$ a high bleeding risk, and $1.7 \%$ contraindications for anticoagulation. Comorbidity was frequent. As for therapy, $82.7 \%$ were taking direct oral anticoagulants (DOAC), $11.0 \%$ vitamin $K$ antagonists (VKA), and $6.3 \%$ no anticoagulant treatment. Anticoagulant therapy was inappropriate in $29.3 \%$ of patients, mainly because DOAC were prescribed without adjusting for age, weight, or serum creatinine and administered without indication according to thromboembolic risk. Only $39.4 \%$ of patients taking VKA were within the therapeutic range. Of all patients receiving DOAC, $48.0 \%$ were taking rivaroxaban, mainly at $20 \mathrm{mg} / \mathrm{d}(73.1 \%)$. Conclusions: Thromboembolic risk is high in geriatric patients with NVAF. Anticoagulation is contraindicated in $<2 \%$ of patients. Oral anticoagulants are prescribed inappropriately in three out of ten patients.

Keywords: Geriatric. Vitamin K antagonists. Anticoagulation. Direct oral anticoagulants. Atrial fibrillation.

\section{Introducción}

La fibrilación auricular (FA) es una arritmia muy frecuente en la práctica clínica habitual. Datos recientes muestran que su prevalencia en adultos se sitúa en torno al 2-4\%, si bien se espera un aumento significativo en los próximos años, principalmente por el envejecimiento de la población ${ }^{1-3}$. La prevalencia de FA se incrementa de manera marcada con la edad. Se estima que a los 55 años uno de cada tres individuos desarrollará FA a lo largo de su vida ${ }^{1,4}$.

De manera global, la FA eleva hasta cinco veces el riesgo de ictus, aunque depende de diferentes características clínicas, como la edad y la presencia de otras comorbilidades ${ }^{1,5,6}$. Aproximadamente en el 20$30 \%$ de todos los ictus isquémicos y en el $10 \%$ de los criptogénicos subyace la FA como causa principal ${ }^{1,5,6}$. Por lo tanto, la prevención adecuada del ictus en los pacientes con FA resulta fundamental ${ }^{1,7,8}$. De hecho, salvo alguna contraindicación específica, se recomienda la anticoagulación para reducir el riesgo de presentar complicaciones tromboembólicas en los pacientes con riesgo elevado de presentar ictus ${ }^{1,7,8}$. Sin embargo, a pesar de esta recomendación, un número significativo de pacientes no se encuentran anticoagulados o no están tomando el tratamiento antitrombótico de manera apropiada, sea por un insuficiente control de la anticoagulación en los que están tomando antagonistas de la vitamina K (AVK) o porque los tratados con anticoagulantes orales de acción directa (ACOD) toman dosis inadecuadas para sus características clínicas ${ }^{9-11}$. Este tratamiento antitrombótico inapropiado ocurre con más frecuencia en las personas de mayor edad, posiblemente por un excesivo miedo al riesgo de hemorragias asociado al tratamiento anticoagulante en esta población, a pesar del riesgo elevado de ictus ${ }^{12-14}$.

Datos del Instituto Mexicano del Seguro Social (IMSS) muestran que la FA representa algo más de una de cada cuatro consultas de urgencias cardiológicas y el $14 \%$ de las altas hospitalarias en los servicios de cardiología ${ }^{15}$. En 2010 casi el 1\% de las altas hospitalarias del sector público sanitario mexicano correspondieron a enfermedad cerebrovascular aguda, y cerca del $44 \%$ eran de causa isquémica, siendo la FA uno de los principales factores subyacentes ${ }^{16}$. De hecho, se estima que en México más de medio millón de personas tienen FA, lo que supone un auténtico problema de salud pública ${ }^{17,18}$. A pesar de la importancia que tiene la FA, existe muy poca información acerca del manejo de la prevención de las complicaciones tromboembólicas en México, sobre todo en la población geriátrica ${ }^{19}$. Este estudio se realizó con el fin de aumentar la información disponible sobre la anticoagulación en el paciente geriátrico con FA en México.

\section{Material y métodos}

El objetivo de este estudio fue determinar los patrones de anticoagulación oral en el adulto > 60 años con fibrilación auricular no valvular (FANV) en el Hospital General Regional N. ${ }^{\circ} 1$ de Querétaro del IMSS. Además, se analizaron las principales comorbilidades asociadas a esta patología, incluyendo el riesgo tromboembólico y hemorrágico y las medicaciones concomitantes, así como el control del índice internacional normalizado (INR) en los pacientes que estaban tomando AVK.

Para ello se realizó un estudio observacional, transversal y retrolectivo en el que se incluyó a sujetos $>60$ años que acudieron a la consulta externa de especialidades del Hospital General Regional N. ${ }^{0} 1$ de Querétaro con el diagnóstico de FANV demostrada por electrocardiograma y corroborada por el médico especialista (cardiología, medicina interna y geriatría) entre el 1 de julio y el 30 de septiembre de 2019. Se excluyó a los pacientes con FA valvular, definida como estenosis mitral de moderada a grave o portador de prótesis 
valvular metálica cardiaca ${ }^{7}$, a pacientes con stent coronario en tratamiento con anticoagulación oral y a pacientes con otras indicaciones de anticoagulación oral diferentes a la FANV, como trombosis venosa profunda, tromboembolia pulmonar, trombofilia primaria 0 secundaria, síndrome antifosfolípido, profilaxis posquirúrgica, etc. Tampoco se incluyó a pacientes con expediente no localizable o incompleto. Se seleccionó a todos los pacientes que acudieron a la consulta externa de cardiología, medicina interna y geriatría durante el periodo de estudio de acuerdo con el listado proporcionado por el sistema informático de las consultas externas (SIMO). El protocolo fue evaluado por el Comité de Ética en Investigación y el Comité Local de Investigación en Salud del Estado de Querétaro del IMSS, obteniendo su autorización con el número de registro R-2019-2201-009. Al ser un estudio transversal, observacional y descriptivo, los investigadores no realizaron ninguna intervención diagnóstica o terapéutica específica sobre los pacientes por su participación en el estudio. En todo momento se respetó la confidencialidad de los pacientes y de los médicos tratantes.

Se analizaron los expedientes clínicos de los pacientes incluidos y se recogieron de manera anonimizada durante la consulta 0 , en el caso de que faltase algún dato, en el momento más próximo a ella, las siguientes variables: datos sociodemográficos (edad, sexo, estado civil, religión y especialidad donde fueron atendidos los pacientes), datos de la exploración física (presión arterial sistólica y frecuencia cardiaca), riesgo tromboembólico (elevado con una puntuación en la escala $\mathrm{CHA}_{2} \mathrm{DS}_{2}$ $\mathrm{VASc}^{20} \geq 2 / 3$ para varón/mujer), riesgo hemorrágico (elevado con una puntuación en la escala HAS-BLED ${ }^{21}$ $\geq 3$; fármacos concomitantes que aumentan el riesgo de hemorragia, como antiagregantes y antiinflamatorios no esteroideos a cualquier dosis; alcoholismo; hemorragia grave previa; insuficiencia hepática, y presión arterial sistólica no controlada), factores de riesgo cardiovascular (hipertensión arterial, diabetes mellitus tipo 2 y tabaquismo), enfermedad vascular (antecedentes de insuficiencia cardiaca, ictus/ataque isquémico transitorio, infarto de miocardio, insuficiencia renal, enfermedad de la aorta y enfermedad arterial periférica), función renal (calculada mediante la tasa de filtrado glomerular por CKD-EPI por medio de la página web de la Sociedad Española de Nefrología ${ }^{22}$ y pruebas complementarias realizadas (en particular el ecocardiograma). Estas variables se determinaron de manera global y según el sexo del paciente. Para el estado civil se consideró la situación estable o permanente en la que se encontraba en relación con sus circunstancias personales y con la legislación (soltero, casado, divorciado, viudo o no especificado). La hemorragia grave se definió como el sangrado por cualquier vía que hubiese condicionado alteraciones hemodinámicas y síndrome anémico agudo o sangrado en el sistema nervioso central u ocular. Un INR lábil se definió como $<60 \%$ del tiempo en rango terapéutico tras 4 semanas de iniciar el tratamiento con AVK según aparecía consignado en el expediente clínico. Respecto al consumo de alcohol, se consideró para el aumento en el riesgo de sangrado la toma de $\geq 8$ copas/semana (equivalente a $40 \mathrm{ml}$ de alcohol puro) ${ }^{21}$.

Se analizó de manera exhaustiva el tratamiento anticoagulante, incluyendo la clase de anticoagulante oral, AVK vs. ACOD y, dentro de estos, el tipo y la dosis. Dentro de los AVK se calculó el porcentaje de pacientes con un INR adecuado (2.0-3.0), así como el de aquellos con un INR $<20>3$. También se estudió si existía alguna contraindicación para la anticoagulación, si esta se realizaba de manera adecuada y la causa de la anticoagulación inapropiada. Estos resultados se analizaron en función del sexo. Asimismo, también se estudió la proporción de pacientes con anticoagulación adecuada de acuerdo con la especialidad que atendía al paciente (cardiología, medicina interna o geriatría). Se consideró indicación adecuada para la anticoagulación una puntuación $\mathrm{CHA}_{2} \mathrm{DS}_{2}$-VASc $\geq 2$ en varones y $\geq 3$ en mujeres, y no adecuada, de 0 en varones y de 1 en mujeres o que no estuviese indicada a pesar de tener una puntuación mayor ${ }^{7}$. Dado el riesgo extremo de sangrado, se consideró contraindicación absoluta para prescribir anticoagulantes orales, a pesar de la presencia de FA con indicación de anticoagulación, que el paciente presentara al menos una de las siguientes situaciones reflejadas en el expediente clínico: diátesis hemorrágicas graves, procesos hemorrágicos activos (úlcera gastrointestinal, neoplasias ulceradas y retinopatía hemorrágica), hemorragia intracraneal en los últimos tres meses, aneurisma intracerebral o hipertensión arterial grave o no controlable. Para definir la dosis correcta del anticoagulante oral se hicieron las siguientes consideraciones, de acuerdo con las guías americanas de $F A^{8}$ :

- Dabigatrán: 150 mg/12 h; en > 75 años, 110 mg/12 h (dosis estándar); evitarlo con tasas de filtrado glomerular $<30 \mathrm{ml} / \mathrm{min}$.

- Apixabán: 5 mg/12 h (dosis estándar); en pacientes con al menos dos de los siguientes criterios: edad $\geq 80$ años, peso corporal $\leq 60 \mathrm{~kg}$ o creatinina sérica $\geq 1.5 \mathrm{mg} / \mathrm{dl}$, dosis de $2.5 \mathrm{mg} / 12 \mathrm{~h}$.

- Rivaroxabán: 20 mg/24 h (dosis estándar); en caso de insuficiencia renal de moderada a grave, 
$15 \mathrm{mg} / 24 \mathrm{~h}$; evitarlo con tasas de filtrado glomerular $<15 \mathrm{ml} / \mathrm{min}$.

- AVK: mantener los niveles de INR entre 2.0 y 3.0. Se definió como subanticoagulado (dosis subóptima) el caso del paciente con un INR $<2$ y sobreanticoagulado (dosis supraóptima) el del que presentaba un INR $>3$.

Se hizo un análisis independiente para los ACOD (dosis no ajustada a edad, peso y creatinina). Para definir la variable prescripción inadecuada se consideró la presencia de una o más de las siguientes condiciones: dosis subóptima o supraóptima de AVK, dosis subóptima de ACOD, dosis de ACOD no ajustada para edad, peso o niveles de función renal, indicación de anticoagulantes en pacientes con puntuación en la escala de $\mathrm{CHA}_{2} \mathrm{DS}_{2}-\mathrm{VASc}<2$ para varones $0<3$ para mujeres y la no indicación de anticoagulantes a pesar del riesgo con una puntuación $\mathrm{CHA}_{2} \mathrm{DS}_{2}-\mathrm{VASc} \geq 2$ para varones $0 \geq 3$ para mujeres.

\section{Análisis estadístico}

Para el cálculo del tamaño de la muestra se consideraron los datos proporcionados por el SIMO de las consultas externas de especialidades del Hospital General Regional N. -1 de Querétaro durante un periodo de tres meses, en los que había unos 300 pacientes de promedio > 60 años con diagnóstico de FANV. Asumiendo una población finita de 300 individuos, una prevalencia esperada del $50 \%$, un poder estadístico del $80 \%$ y un nivel de significación estadística del $5 \%$, se calculó un tamaño muestral mínimo de 106 pacientes para poder contestar de manera adecuada al objetivo principal del estudio. A pesar de ello, dado que se contó con el recurso humano para la recogida de la información, se analizaron todos los expedientes de los pacientes que recibieron atención médica por FANV comprendidos en el periodo de estudio.

Las variables cuantitativas se expresaron como media y desviación típica y las cualitativas como valores absolutos (N) y proporciones (\%). Las variables cuantitativas se compararon mediante la t de Student y las cualitativas se compararon mediante la prueba de chi cuadrada. Se consideró un nivel de significación estadística de 0.05 en todas las pruebas estadísticas. Los datos se analizaron mediante el paquete estadístico SPSS (v25.0).

\section{Resultados}

Se obtuvo el registro de pacientes $>60$ años atendidos con diagnóstico de FANV del 1 de junio al 30 de septiembre de 2019 proporcionado por el SIMO, que incluyó a 300 pacientes. Se localizó el 100\% de los expedientes en el archivo físico y electrónico, se corroboró que se incluyera toda la información requerida y no hubo ninguna exclusión.

Las características clínicas de los pacientes se muestran en la tabla 1. La edad media fue de $77.2 \pm 8.3$ años, y el $53.3 \%$ de los pacientes eran mujeres. La mayoría de los pacientes fueron atendidos en cardiología (81.0\%) y tenían una presión arterial sistólica entre 90 y 139 $\mathrm{mmHg}(87.3 \%)$, una frecuencia cardiaca entre 60 y 100 Ipm $(91.0 \%)$ y un riesgo tromboembólico elevado (91.0\%). En cambio, solo el $22.7 \%$ de los pacientes presentaban un riesgo hemorrágico elevado, el 12.7\% tomaban algún fármaco que aumentaba el riesgo de hemorragia y el $4.3 \%$ tenían antecedentes de hemorragia. La presencia de factores de riesgo cardiovascular fue frecuente, siendo la hipertensión arterial $(72.7 \%)$ y la diabetes mellitus tipo 2 (29.0\%) los más comunes. En cuanto a las enfermedades cardiovasculares asociadas, los antecedentes de insuficiencia renal moderada-grave (42.0\%), insuficiencia cardiaca (25.3\%) e ictus/ ataque isquémico transitorio (13.3\%) fueron las más prevalentes. Respecto a las pruebas complementarias, solo el $12.0 \%$ de los pacientes tenían consignados los hallazgos de ecocardiograma en el expediente.

Se analizaron las características clínicas en función del sexo. Salvo en el estado civil (más viudas: 12.5 vs. $2.1 \%$ ), había menos casos en mujeres con una frecuencia cardiaca $<60 \mathrm{lpm}$ ( 2.5 vs. $7.9 \%$ ), mayor tabaquismo en varones (10.6 vs. $28.6 \%$ ) y mayor edad en el sexo femenino (78.6 \pm 8.1 vs. $75.7 \pm 8.5$ años); el resto de las características fueron similares en ambos grupos (Tabla 1).

En cuanto al tratamiento anticoagulante, el $82.7 \%$ de Ios pacientes estaban tomando ACOD, el $11.0 \%$ AVK y el $6.3 \%$ no tomaban tratamiento anticoagulante. Globalmente, el anticoagulante oral más frecuentemente prescrito fue el rivaroxabán (39.7\%). El $29.3 \%$ de los pacientes estaban tomando anticoagulantes orales de manera inadecuada, siendo las dos principales causas la prescripción de dosis de ACOD no ajustada a edad, peso y nivel de creatinina y la administración de ACOD sin indicación de acuerdo con el riesgo tromboembólico, sin encontrar diferencias en relación con el sexo. Solo se halló contraindicación a la anticoagulación en el $1.7 \%$ de los pacientes de acuerdo con las guías, aunque la indicación siempre se realizó de manera individualizada, tomando en cuenta el riesgo/beneficio. Dos pacientes $(0.7 \%)$ tuvieron presión arterial sistólica $>180$, en quienes se ajustó el tratamiento antihipertensivo (Tabla 2). 
Arch Cardiol Mex. 2022;92(1)

Tabla 1. Características clínicas de la muestra

Características sociodemográficas

\begin{tabular}{|c|c|c|c|c|}
\hline Edad (años) & $77.2 \pm 8.3$ & $78.6 \pm 8.1$ & $75.7 \pm 8.5$ & $0.002^{*}$ \\
\hline $\begin{array}{l}\text { Estado civil, n (\%) } \\
\text { Soltero } \\
\text { Casado } \\
\text { Divorciado } \\
\text { Viudo } \\
\text { No especificado }\end{array}$ & $\begin{array}{c}5(1.7) \\
26(8.7) \\
1(0.3) \\
23(7.7) \\
245(81.6)\end{array}$ & $\begin{array}{c}3(1.9) \\
11(6.9) \\
0 \\
20(12.5) \\
126(78.7)\end{array}$ & $\begin{aligned} 2 & (1.4) \\
15 & (10.8) \\
1 & (0.7) \\
3 & (2.2) \\
119 & (85.0)\end{aligned}$ & 0.01 \\
\hline $\begin{array}{l}\text { Religión, n }(\%) \\
\text { Católica } \\
\text { Cristiana no católica } \\
\text { Otra } \\
\text { Ninguna } \\
\text { No especificado }\end{array}$ & $\begin{aligned} 45 & (15.0) \\
1 & (0.3) \\
1 & (0.7) \\
5 & (1.7) \\
248 & (82.3)\end{aligned}$ & $\begin{array}{c}30(18.7) \\
0 \\
0 \\
3(1.9) \\
127(79.4)\end{array}$ & $\begin{aligned} 15 & (10.7) \\
1 & (0.7) \\
1 & (0.7) \\
2 & (1.4) \\
121 & (86.5)\end{aligned}$ & 0.196 \\
\hline $\begin{array}{l}\text { Especialidad, n (\%) } \\
\text { Cardiología } \\
\text { Geriatría } \\
\text { Medicina Interna }\end{array}$ & $\begin{array}{c}243(81.0) \\
37(12.3) \\
20(6.7)\end{array}$ & $\begin{array}{c}125(78.1) \\
23(14.4) \\
12(7.5)\end{array}$ & $\begin{array}{c}118(84.3) \\
14(10.0) \\
8(5.7)\end{array}$ & 0.393 \\
\hline \multicolumn{5}{|l|}{ Exploración física } \\
\hline $\begin{array}{l}\text { Presión arterial sistólica, } \mathrm{n}(\%) \\
>180 \mathrm{mmHg} \\
140-179 \mathrm{mmHg} \\
90-139 \mathrm{mmHg} \\
<90 \mathrm{mmHg}\end{array}$ & $\begin{array}{c}2(0.7) \\
31(10.3) \\
262(87.3) \\
5(1.7)\end{array}$ & $\begin{array}{c}0(0.0) \\
19(11.9) \\
138(86.2) \\
3(1.9)\end{array}$ & $\begin{array}{c}2(1.4) \\
12(8.6) \\
124(88.6) \\
2(1.4)\end{array}$ & 0.360 \\
\hline $\begin{array}{l}\text { Frecuencia cardiaca, n (\%) } \\
<60 \mathrm{lpm} \\
60-100 \mathrm{Ipm} \\
101-150 \mathrm{Ipm} \\
>150 \mathrm{Ipm}\end{array}$ & $\begin{array}{c}15(5.0) \\
273(91.0) \\
9(3.0) \\
3(1.0)\end{array}$ & $\begin{array}{c}4(2.5) \\
145(90.6) \\
9(5.6) \\
2(1.3)\end{array}$ & $\begin{array}{c}11(7.9) \\
128(91.4) \\
0 \\
1(0.7)\end{array}$ & 0.006 \\
\hline \multicolumn{5}{|l|}{ Riesgo tromboembólico } \\
\hline $\begin{array}{l}\mathrm{CHA}_{2} \mathrm{DS}_{2} \text {-VASc, n (\%) } \\
0 \\
1 \\
2 \\
3 \\
4 \\
5 \\
6 \\
7 \\
8\end{array}$ & $\begin{array}{c}6(2.0) \\
14(4.7) \\
43(14.3) \\
78(26) \\
69(23) \\
48(16) \\
30(10) \\
8(2.7) \\
4(1.3)\end{array}$ & $\begin{array}{c}0 \\
4(2.5) \\
7(4.4) \\
35(21.9) \\
49(30.6) \\
32(20.0) \\
21(13.1) \\
8(5.0) \\
4(2.5)\end{array}$ & $\begin{array}{c}6(4.3) \\
10(7.2) \\
36(25.7) \\
43(30.7) \\
20(14.3) \\
16(11.4) \\
9(6.4) \\
0 \\
0\end{array}$ & 0.725 \\
\hline $\mathrm{CHA}_{2} \mathrm{DS}_{2}$-VASc $\geq 2$ (varón)/ $\geq 3$ (mujer), n (\%) & $273(91.0)$ & $149(93.1)$ & $124(88.5)$ & 0.120 \\
\hline \multicolumn{5}{|l|}{ Riesgo hemorrágico } \\
\hline $\begin{array}{l}\text { HAS-BLED, } n(\%) \\
0 \text { puntos } \\
1 \text { punto } \\
2 \text { puntos } \\
\geq 3 \text { puntos }\end{array}$ & $\begin{array}{c}17(5.7) \\
79(26.3) \\
136(45.3) \\
68(22.7)\end{array}$ & $\begin{array}{c}7(4.4) \\
41(25.6) \\
74(46.3) \\
38(23.7)\end{array}$ & $\begin{array}{l}10(7.2) \\
38(27.1) \\
62(44.3) \\
30(21.4)\end{array}$ & 0.725 \\
\hline $\begin{array}{l}\text { Fármacos, } \mathrm{n}(\%) \\
\text { Aspirina } \\
\text { Clopidogrel } \\
\text { Antiinflamatorios no esteroideos }\end{array}$ & $\begin{array}{l}23(7.7) \\
9(3.0) \\
6(2.0)\end{array}$ & $\begin{array}{l}10(6.2) \\
3(1.9) \\
4(2.5)\end{array}$ & $\begin{array}{l}13(9.3) \\
6(4.3) \\
2(1.4)\end{array}$ & 0.402 \\
\hline Alcoholismo, n (\%) & $39(13.0)$ & $14(8.8)$ & $25(17.9)$ & 0.19 \\
\hline Hemorragia grave previa, n (\%) & $13(4.3)$ & $10(6.2)$ & $3(2.1)$ & 0.081 \\
\hline
\end{tabular}


Tabla 1. Características clínicas de la muestra (Continuación)

\begin{tabular}{|c|c|c|c|c|}
\hline & Total $(n=300,100 \%)$ & Mujeres ( $n=160,53.3 \%$ ) & Varones $(n=140,46.7 \%)$ & $\mathbf{p}$ \\
\hline Insuficiencia hepática, n (\%) & $7(2.3)$ & $4(2.5)$ & $3(2.1)$ & 0.838 \\
\hline INR lábil, n (\%) & $5(1.7)$ & $3(1.9)$ & $2(1.4)$ & 0.763 \\
\hline $\begin{array}{l}\text { Factores de riesgo cardiovascular } \\
\text { Hipertensión arterial, n (\%) } \\
\text { Diabetes tipo 2, n (\%) } \\
\text { Tabaquismo, n (\%) }\end{array}$ & $\begin{array}{l}218(72.7) \\
87(29.0) \\
57(19.0)\end{array}$ & $\begin{array}{l}119(74.4) \\
46(28.8) \\
17(10.6)\end{array}$ & $\begin{array}{l}99(70.7) \\
41(29.3) \\
40(28.6)\end{array}$ & $\begin{array}{l}0.478 \\
0.919 \\
<0.01\end{array}$ \\
\hline $\begin{array}{l}\text { Enfermedad vascular } \\
\text { Insuficiencia cardiaca, } \mathrm{n}(\%) \\
\text { Ictus o ataque isquémico transitorio, } \\
\mathrm{n}(\%) \\
\text { Infarto de miocardio, } \mathrm{n}(\%) \\
\text { Enfermedad de la aorta, } \mathrm{n}(\%)\end{array}$ & $\begin{array}{c}76(25.3 \%) \\
40(13.3) \\
28(9.3) \\
1(0.3)\end{array}$ & $\begin{array}{c}39(24.4 \%) \\
26(16.2) \\
12(7.5) \\
0\end{array}$ & $\begin{array}{c}37(26.4 \%) \\
14(10.0) \\
16(11.4) \\
1(0.7)\end{array}$ & $\begin{array}{l}0.683 \\
0.112 \\
0.243 \\
0.485\end{array}$ \\
\hline \multicolumn{5}{|l|}{ Pruebas complementarias } \\
\hline $\begin{array}{l}\text { Tasa de filtrado glomerular, } \mathrm{n}(\%) \\
<15 \mathrm{ml} / \mathrm{min} \\
15-30 \mathrm{ml} / \mathrm{min} \\
31-60 \mathrm{ml} / \mathrm{min} \\
61-90 \mathrm{ml} / \mathrm{min} \\
>90 \mathrm{ml} / \mathrm{min}\end{array}$ & $\begin{array}{l}3(1.0) \\
10(3.3) \\
113(37.7) \\
142(47.3) \\
32(10.7)\end{array}$ & $\begin{array}{c}1(0.6) \\
5(3.1) \\
56(35.0) \\
82(51.3) \\
16(10.0)\end{array}$ & $\begin{array}{l}2(1.4) \\
5(3.6) \\
57(40.7) \\
60(42.9) \\
16(11.4)\end{array}$ & 0.658 \\
\hline $\begin{array}{l}\text { Ecocardiograma informado en el } \\
\text { expediente, } \mathrm{n}(\%)\end{array}$ & $36(12.0)$ & $18(11.2)$ & $18(12.9)$ & 0.669 \\
\hline
\end{tabular}

De los pacientes que tomaban AVK, el $87.9 \%$ estaban siendo tratados con warfarina y el resto con acenocumarol. Únicamente el $39.4 \%$ de los pacientes anticoagulados con AVK presentaban el INR en rango terapéutico. De los pacientes tratados con ACOD, el $48.0 \%$ estaban tomando rivaroxabán, el $31.4 \%$ apixabán y el $20.6 \%$ dabigatrán. En cuanto a la dosis de ACOD, el $48.7 \%$ de los tratados con apixabán tomaban la dosis estándar ( $5 \mathrm{mg} / 12 \mathrm{~h}$ ), el $73.1 \%$ de los tratados con rivaroxabán también tomaban la dosis estándar (20 mg/día) y en el $96.2 \%$ de los tratados con dabigatrán tomaban $110 \mathrm{mg} / 12 \mathrm{~h}$ (Tabla 2).

En cambio, hubo una diferencia estadísticamente significativa a una anticoagulación más adecuada en los pacientes atendidos en cardiología (72.0\%) y geriatría $(83.7 \%)$ en comparación con los atendidos en medicina interna $(50.0 \%)(p=0,025)$ (Tabla 3$)$.

\section{Discusión}

En este estudio, realizado en una muestra amplia de pacientes de edad avanzada con FANV, el $72 \%$ estaban tomando de manera adecuada anticoagulantes orales.

En comparación con otros estudios realizados en el entorno, en el Registro Mexicano de Fibrilación Auricular (ReMeFa), realizado con el objetivo de conocer las estrategias de manejo (control del ritmo vs. control de la frecuencia cardiaca) en pacientes mexicanos con FA, la edad media fue de 66 años y el $55 \%$ eran mujeres, pero no se reportó el riesgo tromboembólico/ hemorrágico ${ }^{23}$. Más recientemente se ha llevado a cabo el registro CARMEN-AF, que es un estudio observacional, longitudinal y multicéntrico para conocer el empleo de los anticoagulantes orales en pacientes con FANV y al menos un factor de riesgo tromboembólico. Este estudio reclutó a pacientes entre 2014 y 2016 en México con una edad media de 69 años, el $49 \%$ mujeres y el $85.6 \%$ con una puntuación $\mathrm{CHA}_{2} \mathrm{DS}_{2}$ VASc $\geq 2^{24}$. En el registro internacional GARFIELD-AF se reclutó a 4,162 pacientes en Latinoamérica, de los que cerca del $28 \%$ pertenecían a México, con una edad media de 70 años, el $48 \%$ mujeres y el $86.2 \%$ con una puntuación $\mathrm{CHA}_{2} \mathrm{DS}_{2}-\mathrm{VASc} \geq 2^{25}$. Aunque en otros 
Tabla 2. Tratamiento anticoagulante: indicaciones y contraindicaciones

\begin{tabular}{|c|c|c|c|c|}
\hline & $\begin{array}{c}\text { Total } \\
(n=300,100 \%)\end{array}$ & $\begin{array}{c}\text { Mujeres } \\
\text { (n=160, 53.3\%) }\end{array}$ & $\begin{array}{c}\text { Varones } \\
\text { (n=140, } 46.7 \% \text { ) }\end{array}$ & $\mathbf{p}^{*}$ \\
\hline $\begin{array}{l}\text { Anticoagulante, n (\%) } \\
\text { Acenocumarol } \\
\text { Warfarina } \\
\text { Apixabán } \\
\text { Rivaroxabán } \\
\text { Dabigatrán } \\
\text { Sin tratamiento }\end{array}$ & $\begin{array}{c}4(1.3) \\
29(9.7) \\
78(26.0) \\
119(39.7) \\
51(17.0) \\
19(6.3)\end{array}$ & $\begin{array}{c}2(1.2) \\
14(8.8) \\
45(28.1) \\
67(41.9) \\
24(15.0) \\
8(5.0)\end{array}$ & $\begin{array}{c}2(1.4) \\
15(10.7) \\
33(23.6) \\
52(37.1) \\
27(19.3) \\
11(7.9)\end{array}$ & 0.684 \\
\hline $\begin{array}{l}\text { Anticoagulante, } n(\%) \\
\text { AVK } \\
\text { ACOD } \\
\text { Sin tratamiento }\end{array}$ & $\begin{array}{c}281(93.7) \\
33(11.0) \\
248(82.7) \\
19(6.3)\end{array}$ & $\begin{array}{c}152(95.0) \\
16(10.0) \\
136(85.0) \\
8(5.0)\end{array}$ & $\begin{array}{c}129(92.1) \\
17(12.1) \\
112(80.0) \\
11(7.9)\end{array}$ & 0.472 \\
\hline $\begin{array}{l}\text { AVK, } n(\%) \\
\text { Acenocumarol } \\
\text { Warfarina }\end{array}$ & $\begin{array}{c}33(100) \\
4(12.1) \\
29(87.9)\end{array}$ & $\begin{array}{c}16(100) \\
2(12.5) \\
14(87.5)\end{array}$ & $\begin{array}{c}17(100) \\
2(11.8) \\
15(88.2)\end{array}$ & 0.676 \\
\hline $\begin{array}{l}\text { INR, } n(\%) \\
\text { Subanticoagulado INR }<2 \\
\text { En rango terapéutico INR 2-3 } \\
\text { Sobreanticoagulado INR }>3\end{array}$ & $\begin{array}{l}10(30.3) \\
13(39.4) \\
10(30.3)\end{array}$ & $\begin{array}{l}6(37.5) \\
6(37.5) \\
4(25.0)\end{array}$ & $\begin{array}{l}4(23.5) \\
7(41.2) \\
6(35.3)\end{array}$ & 0.655 \\
\hline $\begin{array}{l}\text { Anticoagulantes orales de acción directa, n (\%) } \\
\text { Apixabán } \\
\text { Rivaroxabán } \\
\text { Dabigatrán }\end{array}$ & $\begin{array}{c}248(100) \\
78(31.4) \\
119(48.0) \\
51(20.6)\end{array}$ & $\begin{array}{l}136(100) \\
45(33.1) \\
67(49.3) \\
24(17.6)\end{array}$ & $\begin{array}{l}112(100) \\
33(29.5) \\
52(46.4) \\
27(24.1)\end{array}$ & 0.448 \\
\hline $\begin{array}{l}\text { Dosis de anticoagulantes orales de acción directa, } \mathrm{n}(\%) \\
\text { Apixabán } 5 \mathrm{mg} / 12 \mathrm{~h} \\
\text { Apixabán } 2.5 \mathrm{mg} / 12 \mathrm{~h} \\
\text { Apixabán dosis subóptima } \\
\text { Rivaroxabán } 20 \mathrm{mg} / 24 \mathrm{~h} \\
\text { Rivaroxabán } 15 \mathrm{mg} / 24 \mathrm{~h} \\
\text { Rivaroxabán dosis subóptima }(10 \mathrm{mg} / 24 \mathrm{~h}) \\
\text { Dabigatrán } 110 \mathrm{mg} / 12 \mathrm{~h} \\
\text { Dabigatrán dosis elevada }(150 \mathrm{mg} / 12 \mathrm{~h}) \\
\text { Dabigatrán dosis subóptima }(75 \mathrm{mg} / 12 \mathrm{~h})\end{array}$ & $\begin{array}{c}38(48.7) \\
37(47.4) \\
3(3.9) \\
87(73.1) \\
31(26.1) \\
1(0.8) \\
49(96.2) \\
1(1.9) \\
1(1.9)\end{array}$ & $\begin{array}{c}20(44.4) \\
23(51.1) \\
2(4.5) \\
44(65.7) \\
22(32.8) \\
1(1.5) \\
24(100) \\
0 \\
0\end{array}$ & $\begin{array}{c}18(54.5) \\
14(42.4) \\
1(3.1) \\
43(82.7) \\
9(17.3) \\
0 \\
25(92.6) \\
1(3.7) \\
1(3.7)\end{array}$ & 0.143 \\
\hline $\begin{array}{l}\text { Contraindicación absoluta/relativa, n (\%) } \\
\text { Hemorragia activa } \\
\text { Hemorragia intracraneal reciente } \\
\text { Presión arterial sistólica grave o no controlable } \\
\text { Total de pacientes con contraindicaciones }\end{array}$ & $\begin{array}{l}1(0.3) \\
2(0.7) \\
2(0.7) \\
5(1.7)\end{array}$ & $\begin{array}{l}1(0.6) \\
1(0.6) \\
0(0.0) \\
2(1.2)\end{array}$ & $\begin{array}{l}0(0.0) \\
1(0.7) \\
2(1.4) \\
3(2.1)\end{array}$ & 0.365 \\
\hline Anticoagulación adecuada, n (\%) & $216(72.0)$ & $122(76.3)$ & $94(67.1)$ & 0.080 \\
\hline $\begin{array}{l}\text { Causas de prescripción inadecuada } \\
\text { Causa de anticoagulación inadecuada en pacientes que tomaron AVK } \\
\text { Dosis que condicionó un INR fuera de rango terapéutico } \\
\text { Pacientes que tomaron AVK sin indicación por } \mathrm{CHA}_{2} \mathrm{DS}_{2}-\mathrm{VASc}{ }^{\dagger} \\
\text { Causa de anticoagulación inadecuada en pacientes que tomaron ACOD } \\
\text { Dosis subóptima } \\
\text { Dosis no ajustada a edad, peso o función renal } \\
\text { Pacientes que tomaron ACOD sin indicación por } \mathrm{CHA}_{2} \mathrm{DS}_{2}-\mathrm{VASc}^{\dagger} \\
\text { No anticoagulados a pesar de tener indicación por CHA2DS2-VASc } \\
\text { AVK o ACOD } \\
\text { Anticoagulados a pesar de tener contraindicaciones } \\
\text { AVK } \\
\text { Anticoagulantes directos } \\
\text { Total de prescripciones inadecuadas }\end{array}$ & $\begin{array}{c}\mathrm{n}=33 \\
20(60.6) \\
3(9.0) \\
\mathrm{n}=248 \\
5(2.0) \\
21(8.5) \\
21(8.5) \\
\mathrm{n}=273 \\
16(5.8) \\
\mathrm{n}=2 \\
0 \\
2(100) \\
\mathrm{n}=300 \\
88(29.3)\end{array}$ & $\begin{array}{l}\mathrm{n}=16 \\
10(62.5) \\
1(6.3) \\
\mathrm{n}=136 \\
3(2.2) \\
10(7.4) \\
9(6.6) \\
\mathrm{n}=149 \\
7(4.6) \\
\mathrm{n}=2 \\
0 \\
2(100)^{\Uparrow} \\
\mathrm{n}=160 \\
42(26.2)\end{array}$ & $\begin{array}{c}n=17 \\
10(58.8 \%) \\
2(11.8 \%) \\
n=112 \\
2(1.8 \%) \\
11(9.8 \%) \\
12(10.7 \%) \\
n=124 \\
9(7.2 \%) \\
n=0 \\
0 \\
0(0) \\
n=140 \\
46(32.9)\end{array}$ & $\begin{array}{l}0.829 \\
0.582 \\
\\
0.815 \\
0.478 \\
0.249\end{array}$ \\
\hline
\end{tabular}

*Comparación de las variables cualitativas mediante la prueba chi cuadrada.

tPuntuación de CHA2DS2-VASc $<2$ en varones $y<3$ en mujeres.

łPuntuación de CHA2DS2-VASc $\geq 2$ en varones $y \geq 3$ en mujeres.

§Apixabán $2.5 \mathrm{mg}$ cada $24 \mathrm{~h}$, rivaroxabán $10 \mathrm{mg}$ cada $24 \mathrm{~h}$ y dabigatrán $75 \mathrm{mg}$ cada $12 \mathrm{~h}$

円Pacientes con presión arterial sistólica mayor de $180 \mathrm{mmHg}$ (en la consulta en la que se documentó tensión arterial sistólica > $180 \mathrm{mmHg}$ se les ajustó el tratamiento antihipertensivo).

**Hubo pacientes con más de una indicación prescriptiva inadecuada.

AVK: antagonistas de la vitamina K; ACOD: anticoagulantes orales de acción directa; INR: índice internacional normalizado; $\mathrm{CHA}_{2} \mathrm{DS}_{2}$-VASc: Congestive heart failure (insuficiencia cardíaca congestiva), Hypertension, Age (edad) = 75 años (doblada), Diabetes, Stroke/transient ischemic attack/thromboembolism (ictus, crisis isquémica transitoria o enfermedad tromboembólica (doblada), Vascular disease (enfermedad vascular: antecedente de infarto de miocardio, arteriopatía periférica o placa aórtica), 
Tabla 3. Proporción de pacientes con anticoagulación adecuada de acuerdo con la especialidad que atiende al paciente

\begin{tabular}{|c|c|c|c|c|c|c|}
\hline & & & \multirow[t]{2}{*}{ Total } & \multicolumn{2}{|c|}{ Anticoagulación adecuada } & \multirow[t]{2}{*}{$\mathbf{p}^{*}$} \\
\hline & & & & Sí & No & \\
\hline \multirow[t]{4}{*}{ Especialidad } & Medicina Interna & $\begin{array}{l}\text { Recuento dentro de la } \\
\text { especialidad, } \mathrm{N}(\%)\end{array}$ & $20(100)$ & $10(50.0)$ & $10(50.0)$ & \multirow[t]{3}{*}{0.025} \\
\hline & Cardiología & $\begin{array}{l}\text { Recuento dentro de la } \\
\text { especialidad, N (\%) }\end{array}$ & $243(100)$ & $175(72.0)$ & $68(28.0)$ & \\
\hline & Geriatría & $\begin{array}{l}\text { Recuento dentro de la } \\
\text { especialidad, } \mathrm{N}(\%)\end{array}$ & $37(100)$ & $31(83.7)$ & $6(16.3)$ & \\
\hline & Total & $\begin{array}{l}\text { Recuento dentro de la } \\
\text { especialidad, N (\%) }\end{array}$ & $300(100)$ & $216(72.0)$ & $84(28.0)$ & - \\
\hline
\end{tabular}

*Comparación de las variables cualitativas mediante la prueba chi cuadrada.

países, como España, la edad media de los pacientes con FANV y el riesgo tromboembólico resultan más parecidos a los encontrados en la muestra, ya que se trata de una población más envejecida ${ }^{13,26-28}$, los datos de este estudio aportan una información relevante y novedosa que no ha sido analizada en otros trabajos realizados en México.

En la muestra los pacientes presentaban un elevado número de comorbilidades, lo que es concordante con otros registros ${ }^{13,23-28}$. Esto sin duda no solo añade dificultades en el manejo de estos pacientes, sino que, como señalan las recientes guías de FA de la Sociedad Europea de Cardiología, para mejorar su pronóstico y disminuir la carga de FA son fundamentales el tratamiento adecuado de las comorbilidades (enfermedad vascular, síndrome de apnea del sueño, etc.) y el control de los factores de riesgo cardiovascular ${ }^{1}$.

Se analizaron las características clínicas en función del sexo y se encontró únicamente una mayor edad en las mujeres. Sin embargo, en el registro CARMEN-AF no solo se hallaron diferencias respecto a la edad, sino también en la prevalencia de numerosas comorbilidades $^{24}$. Las diferencias debidas al sexo en la FA son relevantes y no solo incluyen mecanismos, etiología, respuesta al tratamiento y riesgo de ictus, sino también comorbilidades y estrategias de manejo ${ }^{29}$. Probablemente la selección de pacientes en el estudio, de edad avanzada, podría explicar en parte que no se encontraran grandes diferencias respecto al perfil clínico en función del sexo.

Llama la atención que únicamente en el $12 \%$ de los pacientes se había realizado un ecocardiograma, teniendo en cuenta, además, que el $80 \%$ de ellos habían sido atendidos en las consultas de cardiología y que en todo paciente con FA es necesario descartar la presencia de cardiopatía estructural y precisamente el ecocardiograma constituye una prueba básica necesaria para realizar un abordaje más adecuado de estos pacientes ${ }^{1}$.

La proporción de pacientes con contraindicaciones para la anticoagulación no llegó al $2 \%$ de los incluidos en el estudio. Estos resultados confirman que la gran mayoría de pacientes con FANV deberían recibir anticoagulación oral de manera permanente ${ }^{30}$. Sin embargo, hasta un $5.8 \%$ de los pacientes no recibían tratamiento anticoagulante en el estudio. En el registro CARMEN-AF, el $16 \%$ de los pacientes no tomaban ningún tratamiento antitrombótico y el 19\% antiagregantes plaquetarios ${ }^{31}$. En el estudio GARFIELD-AF, en los países reclutados en Latinoamérica estas cifras fueron del 14 y $24 \%$, respectivamente ${ }^{25}$. Tanto el empleo de antiagregantes plaquetarios para la prevención de las complicaciones tromboembólicas como no anticoagular a un paciente geriátrico son graves errores, ya que existe suficiente evidencia para afirmar que, aunque en adultos mayores existe un riesgo incrementado de hemorragia, el de ictus es mucho mayor, y por lo tanto está plenamente justificada la anticoagulación en el paciente geriátrico con FANV ${ }^{32-34}$.

Respecto al tratamiento anticoagulante, alrededor del $83 \%$ de los pacientes estaban tomando ACOD y el $11 \% A V K^{35,36}$. Estas cifras van en línea con lo reportado en otros países de Europa y en EE.UU., donde cada vez es mayor la prescripción de los ACOD frente a los AVK, y con lo recomendado por las guías de práctica clínica ${ }^{1,7,8}$ debido al mejor perfil de eficacia y seguridad de los ACOD en comparación con los AVK ${ }^{37}$.

Un aspecto preocupante del estudio fue que el $28 \%$ de los pacientes estaban tomando de manera inadecuada anticoagulantes orales. En el caso de los AVK, 
solo el $39.4 \%$ de los pacientes presentaban un INR en rango terapéutico, cifra sensiblemente inferior a la reportada en estudios previos $^{13,38}$, lo que se asocia a mayor riesgo de ictus, hemorragia y mortalidad, así como a costes económicos ${ }^{39,40}$. Por lo tanto, se hace necesario el desarrollo de medidas para mejorar el control de la anticoagulación en los pacientes tratados con AVK y, en caso de que no sea posible, plantear el cambio a ACOD ${ }^{1}$. De los pacientes tratados con ACOD, el motivo más frecuente de una incorrecta anticoagulación fue la prescripción de dosis no ajustada a edad, peso y niveles de función renal. A pesar de los grandes beneficios de los ACOD, su dosificación inadecuada podría tener consecuencias clínicas importantes al disminuir la eficacia antitrombótica o aumentar sus efectos tóxicos ${ }^{41,42}$. El rivaroxabán fue el ACOD más frecuentemente prescrito en el estudio y el ACOD en el que se pautó la dosis estándar con mayor frecuencia en términos absolutos, posiblemente por su mayor sencillez de dosificación al depender únicamente de la función renal ${ }^{43}$. Estas diferencias podrían tener influencia en la prevención de las complicaciones tromboembólicas en la práctica clínica ${ }^{44}$. En el estudio no se encontraron diferencias en cuanto al tratamiento anticoagulante respecto al sexo, aspecto que también se ha visto reflejado en el registro CARMEN-AF ${ }^{24}$. Sin embargo, sí se halló una diferencia estadísticamente significativa respecto a una anticoagulación más adecuada en los pacientes atendidos en cardiología y geriatría frente a los tratados en medicina interna. El manejo del paciente geriátrico con FANV supone un reto que exige conocimientos específicos para poder prestar al paciente la atención más individualizada y mejor posible ${ }^{45}$.

El estudio tiene algunas limitaciones. En primer lugar, por su propio diseño, pues sin seguimiento prospectivo ni grupo comparador solo permite generar hipótesis. En segundo lugar, no se estudiaron variables fuera de la indicación (riesgo tromboembólico o hemorragia) que pudieran cambiar la conducta prescriptiva de los médicos, tales como síndromes geriátricos (demencia, polifarmacia, fragilidad, síndrome de caídas o falta de apego al tratamiento, red de apoyo social o familiar, así como capacidad para acudir a los servicios de salud), lo que podría haber motivado que la decisión de prescripción o no estuviera basada en el juicio clínico. Por último, al ser la fuente de información el conjunto de los expedientes clínicos debe considerarse la posibilidad de sesgos en la información registrada en ellos debido a errores en su cumplimentación o a la falta de información, por ejemplo, respecto al tratamiento prescrito por médicos del medio privado. Sin embargo, este tipo de diseño, transversal y observacional, permite conocer de manera más fidedigna cuál es el manejo real en la práctica clínica, ya que la inclusión en él no ha condicionado el manejo del paciente. Además, el tamaño muestral fue lo suficientemente amplio para poder contestar de manera adecuada al objetivo principal del estudio. Por otra parte, al tratarse de un estudio unicéntrico, los resultados solo podrían aplicarse a centros con características similares. No obstante, el tipo de población analizada en el presente trabajo no ha sido estudiada específicamente en México, por lo que los resultados de este estudio aportan una información muy relevante para entender el manejo del paciente geriátrico con FANV en este entorno.

\section{Conclusiones}

Los pacientes geriátricos con FANV tienen un riesgo tromboembólico elevado y numerosas comorbilidades, y menos del $2 \%$ presentan contraindicaciones para anticoagulación. Del total de la muestra, un 5.8\% no recibían tratamiento anticoagulante a pesar de tener indicación, y aproximadamente el $20 \%$ de los pacientes estaban tomando una dosis distinta a la requerida de anticoagulación oral, principalmente por una dosificación no óptima. La mayoría de los pacientes estaban tomando ACOD, siendo el rivaroxabán el más frecuente. Aunque la anticoagulación en el paciente geriátrico supone un reto por su complejidad, existe un amplio margen de mejora, que repercutiría positivamente en la reducción de complicaciones tromboembólicas y hemorrágicas en esta población.

\section{Financiamiento}

La agencia editorial fue financiada por Bayer de México.

\section{Conflictos de interés}

Esta publicación y su contenido son responsabilidad exclusiva de los autores. La asistencia editorial estuvo a cargo de Content Ed Net y fue financiada por Bayer de México.

Los autores no declaran ningún otro conflicto de interés.

\section{Responsabilidades éticas}

Protección de personas y animales. Los autores declaran que los procedimientos seguidos se 
conformaron a las normas éticas del comité de experimentación humana responsable y de acuerdo con la Asociación Médica Mundial y la Declaración de Helsinki.

Confidencialidad de los datos. Los autores declaran que han seguido los protocolos de su centro de trabajo sobre la publicación de datos de pacientes.

Derecho a la privacidad y consentimiento informado. Los autores han obtenido el consentimiento informado de los pacientes y/o sujetos referidos en el artículo. Este documento obra en poder del autor de correspondencia.

\section{Bibliografía}

1. Hindricks G, Potpara T, Dagres N, Arbelo E, Bax JJ, Blomström-Lundqvist C, et al.; ESC Scientific Document Group. 2020 ESC Guidelines for the diagnosis and management of atrial fibrillation developed in collaboration with the European Association of Cardio-Thoracic Surgery (EACTS). Eur Heart J. 2021;42(5):373-498.

2. Benjamin EJ, Muntner P, Alonso A, Bittencourt MS, Callaway CW, Carson AP, et al.; American Heart Association Council on Epidemiology and Prevention Statistics Committee and Stroke Statistics Subcommittee. Heart disease and stroke statistics - 2019 update: a report from the American Heart Association. Circulation. 2019;139(10):e56-528.

3. Krijthe BP, Kunst A, Benjamin EJ, Lip GY, Franco OH, Hofman A, et al. Projections on the number of individuals with atrial fibrillation in the European Union, from 2000 to 2060. Eur Heart J. 2013;34(35):2746-51.

4. Staerk L, Wang B, Preis SR, Larson MG, Lubitz SA, Ellinor PT, et al. Lifetime risk of atrial fibrillation according to optimal, borderline, or elevated levels of risk factors: cohort study based on longitudinal data from the Framingham Heart Study. BMJ. 2018;361:k1453.

5. Pisters R, Lane DA, Marin F, Camm AJ, Lip GY. Stroke and thromboembolism in atrial fibrillation. Circ J. 2012;76(10):2289-304.

6. Hart RG, Diener HC, Coutts SB, Easton JD, Granger CB, O'Donnell MJ, et al. Cryptogenic Stroke/ESUS International Working Group. Embolic strokes of undetermined source: the case for a new clinical construct. Lancet Neurol. 2014;13(4):429-38.

7. Kirchhof P, Benussi S, Kotecha D, Ahlsson A, Atar D, Casadei B, et al. 2016 ESC Guidelines for the management of atrial fibrillation developed in collaboration with EACTS. Eur Heart J. 2016;37(38):2893-962.

8. January CT, Wann LS, Calkins H, Chen LY, Cigarroa JE, Cleveland JC Jr, et al. 2019 AHA/ACC/HRS focused update of the 2014 AHA/ACC/HRS Guideline for the Management of Patients With Atrial Fibrillation: A report of the American College of Cardiology/American Heart Association Task Force on Clinical Practice Guidelines and the Heart Rhythm Society. J Am Coll Cardiol. 2019;74(1):104-32.

9. Vallakati A, Lewis WR. Underuse of anticoagulation in patients with atrial fibrillation. Postgrad Med. 2016;128(2):191-200.

10. Henrard S, Vandenabeele C, Marien S, Boland B, Dalleur O. Underuse of anticoagulation in older patients with atrial fibrillation and CHADS Score $\geq 2$ : Are we doing better since the marketing of direct oral anticoagulants? Drugs Aging. 2017;34(11):841-50.

11. Escobar C, Barrios V, Prieto L. Therapeutic behavior of primary care physicians in patients with atrial fibrillation taking vitamin $\mathrm{K}$ antagonists not adequately controlled. Eur J Intern Med. 2016;30:e17-8.

12. Chao TF, Liu CJ, Lin YJ, Chang SL, Lo LW, Hu YF, et al. Oral anticoagulation in very elderly patients with atrial fibrillation: a nationwide cohort study. Circulation. 2018;138(1):37-47.

13. Gullón A, Suárez C, Díez-Manglano J, Formiga F, Cepeda JM, Pose A, et al.; investigadores del estudio NONAVASC. Características y tratamiento antitrombótico de los pacientes ancianos con fibrilación auricular no valvular hospitalizados en Medicina Interna. Registro NONAVASC. Med Clin (Barc). 2017;148(5):204-10.

14. Fernández CS, Gullón A, Formiga $F$. The problem of underdosing with direct-acting oral anticoagulants in elderly patients with nonvalvular atrial fibrillation. J Comp Eff Res. 2020;9(7):509-23.

15. Moreno-Ruiz LA, Rodríguez-Piña H, Martínez-Flores JE. Fibrilación auricular. Evaluación y tratamiento. Rev Med Inst Mex Seguro Soc. 2012; 50(3):273-84

16. Chiquete E, Ruiz-Sandoval JL, Murillo-Bonilla LM, Arauz A, Villarreal-Careaga J, León-Jiménez $C$, et al. Egresos por enfermedad vascular cerebral aguda en instituciones públicas del sector salud de México: Un análisis de 5.3 millones de hospitalizaciones en 2010. Rev Mex Neuroci. 2012;13:252-8.
17. Rodríguez-Diez $G$, Márquez MF, Iturralde-Torres $P$ Molina-Fernández de LLG, Pozas-Garza G, Cordero-Cabra A, et al. Joint Mexican position document on the treatment of atrial fibrillation. Arch Cardiol Mex. 2020;90(1):69-76.

18. Stevens B, Pezzullo L, Verdian L, Tomlinson J, Estrada-Aguilar C, George $A$, et al. La carga económica de cuatro condiciones cardiacas en México: hipertensión; insuficiencia cardiaca; infarto de miocardio y fibrilación auricular. Arch Cardiol Mex. 2018;88(3):241-4

19. González-Hermosillo JA, Márquez MF, Ocampo-Peña S; Comité Cientíico del Registro CARMEN-AF. Diseño de un registro de fibrilación auricular y riesgo embólico en México: CARMEN-AF. Arch Cardiol Mex. 2017;87(1):5-12

20. Lip GY, Nieuwlaat R, Pisters R, Lane DA, Crijns HJ. Refining clinical risk stratification for predicting stroke and thromboembolism in atrial fibrillation using a novel risk factor-based approach: the euro heart survey on atrial fibrillation. Chest. 2010;137(2):263-72.

21. Pisters R, Lane DA, Nieuwlaat R, de Vos CB, Crijns HJ, Lip GY. A novel user friendly score (HAS-BLED) to assess 1-year risk of major bleeding in patients with atrial fibrillation: the Euro Heart Survey. Chest. 2010;138(5):1093-100.

22. Calculadora de función renal. Estimación del filtrado glomerular MDR/ CKD-EPI [Internet]. Sociedad Española de Nefrología. Disponible en: https://www.senefro.org/modules.php?name=calcfg

23. Lara-Vaca S, Cordero-Cabra A, Martínez-Flores E, Iturralde-Torres $P$. Registro Mexicano de Fibrilación Auricular (ReMeFa). Gac Med Mex. 2014;150(Suppl 1):48-59.

24. González-Hermosillo JA, Baños-González MA, Guevara-Valdivia ME, Vázquez-Acosta JA, de Los Ríos Ibarra MO, Aguilar-Linares KA, et al.; CARMEN-AF Committees and Investigators. Gender differences and management of stroke risk of nonvalvular atrial fibrillation in an upper middle-income country: Insights from the CARMEN-AF registry. Int $\mathrm{J}$ Cardiol Heart Vasc. 2019;22:117-22.

25. Jerjes-Sánchez C, Corbalán R, Barretto ACP, Luciardi HL, Allu J, Illingworth L, et al.; GARFIELD-AF Investigators. Stroke prevention in patients from Latin American countries with non-valvular atrial fibrillation: Insights from the GARFIELD-AF registry. Clin Cardiol. 2019;42(5):553-60.

26. Mostaza JM, Jiménez MJR, Laiglesia FJR, Peromingo JAD, Robles MB Sierra EG, et al. Clinical characteristics and type of antithrombotic treatment in a Spanish cohort of elderly patients with atrial fibrillation according to dependency, frailty and cognitive impairment. J Geriatr Cardiol. 2018;15(4):268-74

27. Barrios V, Escobar C, Calderón A, Zamorano JL. Prevalence of renal dysfunction according to the type of atrial fibrillation and anticoagulation treatment in patients who attended primary care in Spain. Future Cardiol. 2014;10(2):215-20.

28. Cerdá M, Cerezo-Manchado JJ, Johansson E, Martínez F, Fernández M, Varela A, et al. Facing real-life with direct oral anticoagulants in patients with nonvalvular atrial fibrillation: outcomes from the first observational and prospective study in a Spanish population. J Comp Eff Res. 2019;8(3):165-78.

29. Westerman S, Wenger N. Gender differences in atrial fibrillation: A review of epidemiology, management, and outcomes. Curr Cardiol Rev. 2019;15(2):136-44.

30. Barrios V, Escobar C, Calderón A, Rodríguez Roca GC, Llisterri JL, Polo García J. Uso del tratamiento antitrombótico según la escala $\mathrm{CHA}_{2} \mathrm{DS}_{2}$ VASc en los pacientes con fibrilación auricular en atención primaria. Rev Esp Cardiol (Engl Ed). 2014;67(2):150-1.

31. Márquez MF, Baños-González MA, Guevara-Valdivia ME, Vázquez-Acosta J, de los Ríos Ibarra MO, Aguilar-Linares JA, et al. Anticoagulation therapy by age and embolic risk for nonvalvular atrial fibrillation in Mexi$\mathrm{co}$, an upper-middle-income country: The CARMEN-AF Registry. Glob Heart. 2020;15(1):32.

32. Mant J, Hobbs FD, Fletcher K, Roalfe A, Fitzmaurice D, Lip GY, et al.; BAFTA investigators, Midland Research Practices Network (MidReC). Warfarin versus aspirin for stroke prevention in an elderly community population with atrial fibrillation (the Birmingham Atrial Fibrillation Treatment of the Aged Study, BAFTA): a randomised controlled trial. Lancet. 2007;370(9586):493-503.

33. Alnsasra H, Haim M, Senderey AB, Reges O, Leventer-Roberts M, Arnson $Y$, et al. Net clinical benefit of anticoagulant treatments in elderly patients with nonvalvular atrial fibrillation: experience from the real world. Heart Rhythm. 2019;16(1):31-7.

34. Sardar P, Chatterjee S, Chaudhari S, Lip GY. New oral anticoagulants in elderly adults: evidence from a meta-analysis of randomized trials. J Am Geriatr Soc. 2014;62(5):857-64

35. Cowan JC, Wu J, Hall M, Orlowski A, West RM, Gale CP. A 10 year study of hospitalized atrial fibrillation-related stroke in England and its association with uptake of oral anticoagulation. Eur Heart J. 2018;39(32):2975-83.

36. Katz DF, Maddox TM, Turakhia M, Gehi A, O'Brien EC, Lubitz SA, et al. Contemporary trends in oral anticoagulant prescription in atrial fibrillation patients at low to moderate risk of stroke after guideline-recommended

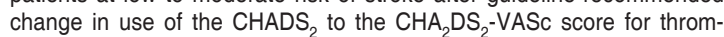
boembolic risk assessment: Analysis from the National Cardiovascular Data Registry's Outpatient Practice Innovation and Clinical Excellence Atrial Fibrillation Registry. Circ Cardiovasc Qual Outcomes. 2017; 10(5):e003476. 
Arch Cardiol Mex. 2022;92(1)

37. Ruff CT, Giugliano RP, Braunwald E, Hoffman EB, Deenadayalu N Ezekowitz MD, et al. Comparison of the efficacy and safety of new ora anticoagulants with warfarin in patients with atrial fibrillation: a meta-analysis of randomised trials. Lancet. 2014;383(9921):955-62.

38. Barrios V, Escobar C, Prieto L, Osorio G, Polo J, Lobos JM, et al. Control de la anticoagulación en pacientes con fibrilación auricular no valvular asistidos en atención primaria en España. Estudio PAULA. Rev Esp Cardiol (Engl Ed). 2015;68(9):769-76.

39. Haas S, Ten Cate H, Accetta G, Angchaisuksiri P, Bassand JP, Camm AJ et al. Quality of vitamin K antagonist control and 1-year outcomes in patients with atrial fibrillation: A global perspective from the GARFIELD-AF Registry. PLoS One. 2016;11(10):e0164076.

40. Briere JB, Bowrin K, Wood R, Holbrook T, Roberts J. The cost of warfarin treatment for stroke prevention in patients with non-valvular atrial fibrillation in Mexico from a collective perspective. J Med Econ. 2017;20(3):266-72.

41. Ruiz Ortiz $M$, Muñiz J, Raña Míguez $P$, Roldán I, Marín $F$, Esteve-Pastor MA, et al. Inappropriate doses of direct oral anticoagulants in real-world clinical practice: prevalence and associated factors. A subanalysis of the FANTASIIA Registry. Europace. 2018;20(10):1577-83.
42. Steinberg BA, Shrader P, Pieper K, Thomas L, Allen LA, Ansell J, et al.; Outcomes Registry for Better Informed Treatment of Atrial Fibrillation (ORBIT-AF) II Investigators. Frequency and outcomes of reduced dose non-vitamin $\mathrm{K}$ antagonist anticoagulants: results from ORBIT-AF II (the Outcomes Registry for Better Informed Treatment of Atrial Fibrillation II). J Am Heart Assoc. 2018;7(4):e007633.

43. Steffel J, Verhamme P, Potpara TS, Albaladejo P, Antz M, Desteghe L, et al. The 2018 European Heart Rhythm Association Practical Guide on the use of non-vitamin K antagonist oral anticoagulants in patients with atrial fibrillation. Eur Heart J. 2018;39(16):1330-93.

44. Escobar C, Martí-Almor J, Pérez Cabeza A, Martínez-Zapata MJ. Anticoagulantes orales directos frente a antagonistas de la vitamina $\mathrm{K}$ en pacientes con fibrilación auricular de la práctica clínica: revisión sistemática y metanálisis. Rev Esp Cardiol (Engl Ed). 2019;72(4):305-16.

45. Alcocer $L$. Challenges and treatment for stroke prophylaxis in patients with atrial fibrillation in Mexico: A review. Am J Cardiovasc Drugs. 2016;16(3):171-82. 